\title{
High-throughput isolation of recombinant antibodies against recombinant allergens
}

\author{
Claudio Rhyner ${ }^{1}$, Zoltan Konthur ${ }^{2}$, Kurt Blaser ${ }^{1}$, and Reto Crameri ${ }^{1}$ \\ ${ }^{1}$ Swiss Institute of Allergy and Asthma Research, Davos, Switzerland and \\ ${ }^{2}$ Max Planck Institute of Molecular Genetics, Berlin, Germany
}

BioTechniques 35:672-674 (October 2003)

Immunoglobulin $\mathrm{E}$ (IgE)-mediated allergic diseases have become a serious health problem in industrialized countries $(1,2)$. Routine diagnosis of allergic conditions is based on clinical history, skin test reactivity, and serum IgE determinations against the offending agents (3). Both skin test reactivity and determinations of serum IgE levels reflect directly on the quality of the allergen extracts used (4). Depending on the exact content of a single allergen in the extract and on the sensitization pattern of the patient, discrepant diagnostic results can be obtained using commercial allergen preparations. Unfortunately, most of the extracts used for diagnosis are not standardized $(5,6)$. The biochemical nature and content of the allergenic components are unknown; therefore, the allergenicity of different extracts cannot be compared. The application of modern molecular biology techniques in allergology allowed for the cloning and molecular characterization of major and minor allergens (7) shown to be powerful reagents for the in vitro and in vivo diagnosis of allergic conditions (4). However, in the majority of cases, reagents allowing for the quantification of individual allergens in commercial allergenic products are still lacking. Monospecific antibodies raised against allergens would substantially facilitate improved quality control and standardization of allergen extracts and lead to the development of more consistent products for clinical use $(6,8)$.

Recombinant immunoglobulin gene libraries cloned in phage or phagemid vectors are an in vitro simulation of antibody repertoires, allowing for the production of antibodies in prokaryotic hosts without immunization or the use of animals (9). The fast-increasing number of recombinant allergens and the need for sensitive reagents for their detection prompted us to implement high-throughput technologies (10) for the rapid selection of allergen-specific antibodies.

Starting from "single pot" naive human antibody libraries that have been previously described (11), we performed parallel selections of phage carrying functional, binding single-chain fragment variables (scFvs) against different recombinant allergens.

Phage antibody libraries were processed and enriched using a prototype pin-based magnetic particle processor (ThermoLabsystems, Helsinki, Finland) combined with liquid-handling robots (KingFisher ${ }^{\circledR}$; ThermoLabsystems) (10). The magnetic particle processor is an automated device that enables the parallel handling of up to 96 individual screenings using 96 magnetic pins that correspond to the position of a 96-well microplate. The magnetic particle processor has a head that consists of two slide-in holders in standard microplate format that are equipped with up to 96 pin-based permanent magnets in rows of eight and the corresponding number of polypropylene caps. The head can be moved along the $\mathrm{x}-, \mathrm{y}-$ and $\mathrm{z}$-axis (up and down). Moving the whole head along the y-axis, the magnets covered with the plastic caps can be inserted into a prefilled plate containing magnetic particles that are attracted and hold in position until the magnets are pulled from the caps. By moving the head along the $\mathrm{x}$-axis, the particles can be transferred to new plates for washing steps and finally resuspended by pulling the magnets from the caps. Every movement of the head and slide-in holders is software-driven and can be controlled with respect to speed, position, and time by user-defined protocols.
Different recombinant allergens (Table 1) were expressed in Escherichia coli and affinity-purified by immobilized metal affinity chromatography (IMAC) (12). Prior to loading, magnetic particles $[10 \mu \mathrm{L} / 250 \mu \mathrm{g}$ nickel-nitrilotriacetic acid (Ni-NTA) Silica Beads; Qiagen, Hilden, Germany] were washed twice for $10 \mathrm{~min}$ in PBST [phosphate-buffered saline (PBS), $0.1 \%$ Tween ${ }^{\circledR} 20$ (Fluka, Buchs, Switzerland)] and subsequently loaded by incubation for $1 \mathrm{~h}$ at room temperature in $200 \mu \mathrm{L}$ purified protein $(100 \mu \mathrm{g} / \mathrm{mL}$ in PBS). Allergen-loaded magnetic particles were washed twice in PBST, and the remaining free binding sites were blocked for $1 \mathrm{~h}$ at room temperature with PTM (PBS, $1 \%$ Tween 20 , and $2 \%$ skimmed milk powder). Blocked, protein-loaded magnetic particles and scFv-carrying phage were co-incubated for $1 \mathrm{~h}$ at room temperature and subjected to 20 automated washing steps using the robotic device to decrease unspecific phage. After washing, the particles carrying bound phage were released into microplate wells containing $200 \mu \mathrm{L} \log$ phase E. coli TG1 cells and incubated for $30 \mathrm{~min}$ at room temperature for infection. Phage titration was performed as previously described (13), and the plates were shaken overnight at $37^{\circ} \mathrm{C}$ to generate phage for further rounds of affinity selection. After 4-5 rounds, affinity-enriched phage were subjected to further investigation.

Phage ELISAs were performed in MaxiSorp ${ }^{\circledR}$ ImmunoPlates (Nalge Nunc International, Roskilde, Denmark). Recombinant allergens (400 ng in 150 $\mu \mathrm{L}$ PBS/well, $\mathrm{pH}$ 7.4) were coated overnight at $4^{\circ} \mathrm{C}$ or $2 \mathrm{~h}$ at room temperature. Blocking was performed with PTM for at least $3 \mathrm{~h}$ at room temperature or at $4^{\circ} \mathrm{C}$ overnight. After washing with PBST, $10^{10} \mathrm{scFv}$-carrying phagemid particles [colony-forming unit (cfu)] in $150 \mu \mathrm{L}$ PTM were applied to the first well and serially diluted at a 1:1 ratio throughout the plate. Alternatively, the allergens were diluted in the same way, starting from $1 \mu \mathrm{g}$ protein in $150 \mu \mathrm{L}$ PBS applied in the first well. In these experiments, the amount of scFv phage particles was kept constant (approximately $5 \times 10^{9} \mathrm{cfu} /$ well). After washing, bound phage was detected with a horseradish peroxidase 
Table 1. Overview of Recombinant Allergens Used for Enrichment

\begin{tabular}{|c|c|c|c|c|c|c|}
\hline Allergen & Organism & Function $^{a}$ & $\begin{array}{c}m \\
(k D a)^{a}\end{array}$ & $\begin{array}{c}\text { GenBank }^{\circledR} \\
\text { Accession No. }^{a}\end{array}$ & scFv Clones & ELISA $^{b}$ \\
\hline Asp $f 1$ & Aspergillus fumigatus & Ribotoxin & 16.8 & S39330 & $1-1,1-7,1-11$ & 1.6 \\
\hline Asp f 4 & A. fumigatus & Unknown & 30.0 & AJ001732 & $4-3,4-11$ & 0.4 \\
\hline Asp f 8 & A. fumigatus & P2 protein & 11.1 & AJ224333 & $8-1,8-4,8-6$ & 1.4 \\
\hline Asp f 9 & A. fumigatus & Unknown & 32.3 & AJ223327 & $9-9,9-11$ & 0.6 \\
\hline Asp f 11 & A. fumigatus & Cyclophilin & 19.6 & AJ006689 & $11-4$ & 0.8 \\
\hline Asp f 15 & A. fumigatus & $\begin{array}{c}\text { Putative serine } \\
\text { protease }\end{array}$ & 15.9 & AJ002026 & $\begin{array}{l}15-2,15-3 \\
15-5,15-9\end{array}$ & 1.2 \\
\hline $\begin{array}{l}\text { scFv, sing } \\
\text { aAccording } \\
\text { bAbsorbar } \\
\text { unrelated }\end{array}$ & $\begin{array}{l}\text {-chain fragment variable } \\
\text { to Reference } 11 . \\
\text { e values of the selected } \\
\text { Asp f } 1 \text { allergen and was }\end{array}$ & $\begin{array}{l}\text { n.d., not determined. } \\
\text { olyclonal phage populatic } \\
<0.05 \text { in all cases. }\end{array}$ & Backgr & d absorption was & rmined agains & \\
\hline
\end{tabular}

(HRP)-conjugated anti-M13 monoclonal antibody (Amersham Biosciences, Buckinghamshire, UK) diluted 1/5000 in PTM (1 $\mathrm{h}$ at room temperature) and visualized with $2^{\prime}, 2^{\prime}$-azino-bis(3-ethylbenz-thiazoline-6-sulphonic acid) diammonium salt (Sigma, St. Louis, MO, USA) in $0.05 \mathrm{M}$ citric acid, following the manufacturer's recommendations. Developed plates were read in a Vmax ${ }^{\circledR}$ ELISA Microplate Reader (Molecular
Devices, Sunnyvale, CA, USA) at 410 $\mathrm{nm}$. Parallel high-throughput selection of $\mathrm{scFv}$-displaying phage from a human naive repertoire library was performed using 10 recombinant allergens that had been derived from different sources (Table 1). In eight cases $(80 \%)$, the polyclonal enrichment of allergen-specific scFv-displaying phage was obtained, and the selected scFv mixtures were shown to bind concentration depend-

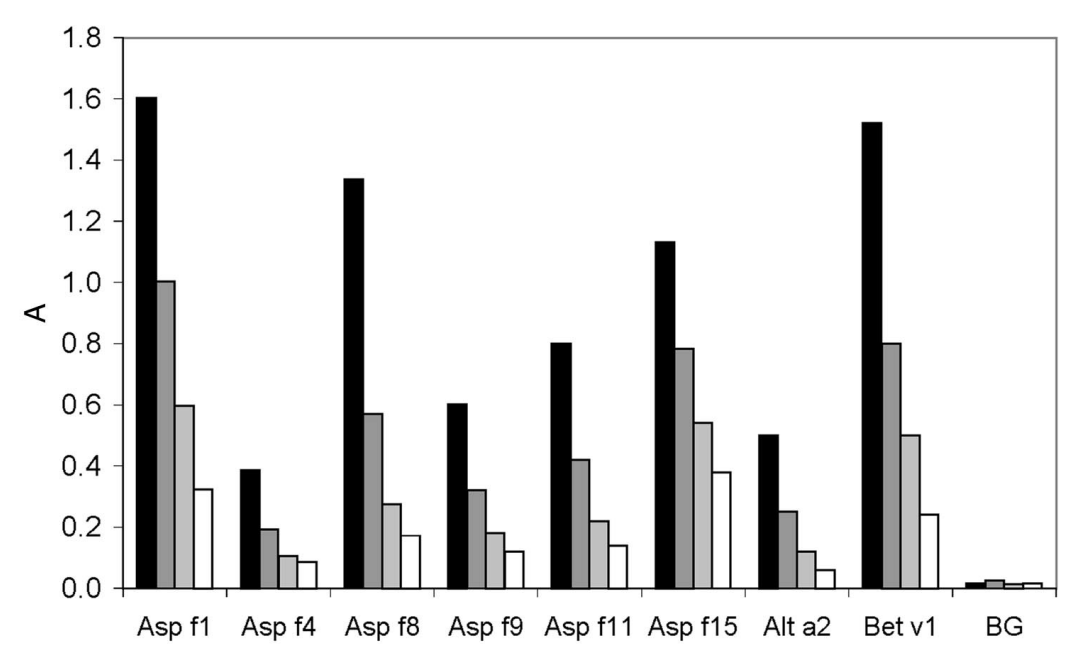

Figure 1. ELISA assays with 2-fold serially diluted enriched polyclonal phage against different recombinant allergens. BG represents the background obtained with phage from the unselected library to the solid phase coated rAsp f 15 allergen. Similar background values were obtained with all allergens (data not shown).

ently to the allergen used for selection in ELISA (Figure 1), indicating a high rate of success confirmed by ELISA analyses of monospecific phage-rescued randomly picked colonies (Table 1). Simple binding to plastic surfaces or blocking agents was excluded, which illustrated a concentration-dependent decrease of the absorbance values in ELISA, where the wells were coated with decreasing amounts of allergen diluted 1:1 throughout the plate, starting from $1 \mu \mathrm{g}$ protein applied in the first well. In these experiments, the amount of $\mathrm{scFv}$ phage particles was kept constant $\left(5 \times 10^{9} \mathrm{cfu} /\right.$ well; data not shown).

For inhibition experiments, a constant amount of phage $\left(5 \times 10^{9} \mathrm{cfu}\right)$ were preincubated with soluble recombinant allergen in decreasing concentrations in a separate, blocked ELISA plate for $30 \mathrm{~min}$ at room temperature. After this preincubation step, the phageallergen mixture was transferred to blocked ELISA plates coated with 400 ng recombinant allergen per well and processed as described earlier.

Inhibition experiments were performed with polyclonal phage (data not shown) as well as monoclonal phage to confirm allergen specificity. Figure 2 shows that for $\mathrm{ScFv}$ phage raised against rAsp $\mathrm{f} 15$, increasing allergen concentra- 


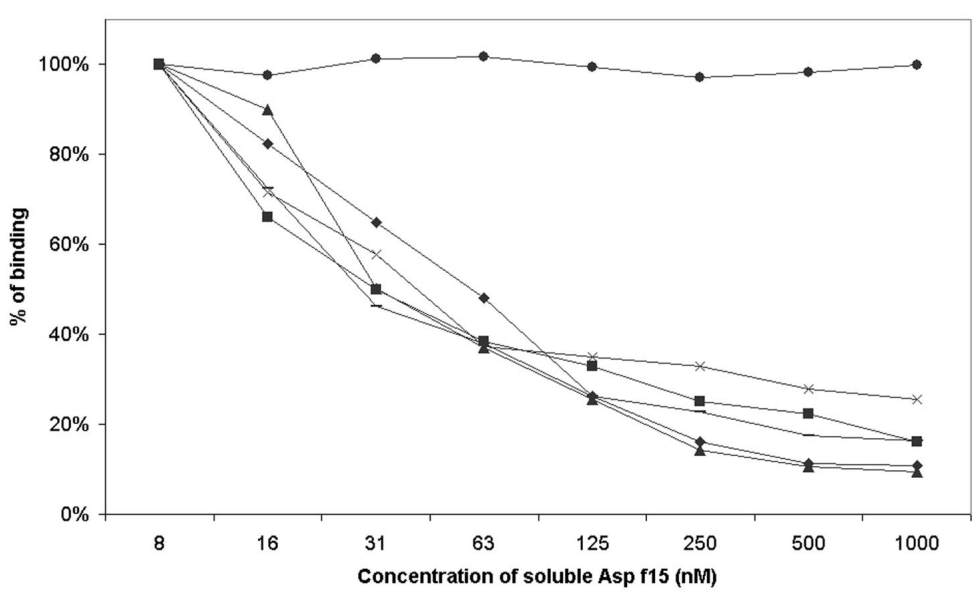

Figure 2. Specific inhibition of phage binding to solid phase coated antigen by increasing concentrations of antigen in fluid phase. Competition ELISA of Asp $\mathrm{f} 15$-specific single clone phage $(\bullet, \mathbf{\square}$, $\boldsymbol{\Delta}, \times)$, polyclonal Asp f 15-specific phage population (-), and as a negative control, the polyclonal Asp f 15 phage population

tions in fluid phase reduce specific binding of the scFv-displaying phage to solid phase coated allergen in a concentrationdependent manner, which demonstrates the specificity of the antigen-antibody interaction. $\mathrm{rAsp} \mathrm{f} 1$, an unrelated protein/ allergen used as a negative control, was unable to influence the binding capacity of the rAsp f 15-specific phage, further confirming the specificity of the reaction.

In summary, we showed the rapid selection of allergen-specific scFv from naive human antibody libraries in a highthroughput format. The validation of the enriched libraries in polyclonal solid phase ELISA and ELISA inhibition experiments showed the specificity of the selected phage populations for their targets in 8 of 10 cases (Figure 1) confirmed at the single clone level, as exemplified for rAsp f 15-specific phage in Figure 2. These results showed that combinatorial antibody libraries displayed on the phage surface are suitable for the rapid isolation of specific binders against virtually any allergen, as also shown for other antigens (9). The amplification and screening of phage surface displayed antibody libraries performed at high-throughput using pin-based magnetic particle processors will facilitate the characterization, quality control, and standardization of allergen extracts, which will lead to the development of more consistent products for clinical use. However, the use of automated magnetic particle processor technology for the selection of antigen- specific phage has several advantages current state of development, the automated setup enables the simultaneous selection of up to 96 samples, in which phage selection and amplification between selection rounds are performed in microplates. Except for the prefilling of microplates, the technique does not involve liquid handling or user intervention because all washing and incubation conditions can be reproducibly customized independently of experimental skills. Finally, the magnetic particle processor is pin-based and modular and can therefore be easily integrated into larger robotic solutions based on the same principle (14), enabling the industrial screening of high-density arrays to rapidly generate oligoclonal mixtures of target-binding molecules.

\section{ACKNOWLEDGMENTS}

Human scFv phage display libraries were kindly provided by I. M. TomlinMolecular Biology, Cambridge, UK). We are grateful to Prof. M. Breitenbach for the gift of rAlt a 2 protein and to Prof. P. Rösch for the gift of the cDNA encoding Bet $v 1$. We are grateful to Prof. H. Lehrach for continuous support. This work was supported by the Swiss National Science Foundation grant no. 31-063381.00 (to R.C.). compared to manual screening. At the son and $G$. Winter (MRC Laboratory of

\section{REFERENCES}

1.Howarth, P.H. 1998. Is allergy increasing?early life influences. Clin. Exp. Allergy 28(Suppl 6):2-7.

2.Smith, D.H., D.C. Malone, K.A. Lawson, L.J. Okamoto, C. Battista, and W.B. Saunders. 1997. A national estimate of the economic costs of asthma. Am. J. Respir. Crit. Care Med. 156: 787-793.

3.Eriksson, N.E. 1994. Diagnosis of IgE mediated allergy in clinical practise. Allergol. Immunopathol. (Madr) 22:139-151.

4.Schmid-Grendelmeier, P. and R. Crameri. 2001. Recombinant allergens for skin testing. Int. Arch. Allergy Immunol. 125:96-111.

5.Nielsen, N.H., A. Dirksen, H. Mosbech, J. Launbjerg, I. Biering, and M. Soborg. 1992. Skin prick testing with standardized extracts from 3 different manufacturers. A comparative randomized study. Allergol. Immunopathol. (Madr) 20:246-248.

6.Vailes, L., S. Sridhara, O. Cromwell, B. Weber, M. Breitenbach, and M. Chapman. 2001. Quantitation of the major fungal allergens, Alt a 1 and Asp $\mathrm{f} 1$, in commercial allergenic pruducts. J. Allergy Clin. Immunol. 107:641-646.

7.King, T.P., D. Hoffman, H. Lowenstein, D.G. Marsh, T.A. Platts-Mills, and W. Thomas. 1995. Allergen nomenclature. Allergy 50:765774

8.Aalberse, R.C. and R. Van Ree. 2000. Monoclonal antibody assays for allergens: pick your antibodies with care! J. Allergy Clin. Immunol. 106:625-626.

9.Hoogenboom, H.R. and P. Chames. 2000 Natural and designer binding sites made by phage display technology. Immunol. Today 21: 371-378.

10.Walter, G., Z. Konthur, and H. Lehrach. 2001 High-throughput screening of surface displayed gene products. Comb. Chem. High Throughput Screen. 4:193-205.

11.de Wildt, R.M., C.R. Mundy, B.D. Gorick, and I.M. Tomlinson. 2000. Antibody arrays for high-throughput screening of antibody-antigen interactions. Nat. Biotechnol. 18:989-994.

12.Crowe, J., H. Dobeli, R. Gentz, E. Hochuli, D. Stuber, and K. Henco. 1994. 6×His-NiNTA chromatography as a superior technique in recombinant protein expression/purification. Methods Mol. Biol. 31:371-387.

13.Crameri, R., G. Achatz, M. Weichel, and C. Rhyner. 2002. Direct selection of cDNAs by phage display. Methods Mol. Biol. 185:461-469.

14.Maier, E., S. Meier-Ewert, D. Bancroft, and H. Lehrach. 1997. Automated array technology for gene expression profiling. Drug Discov. Today 2: 315-324.

Received 10 March 2003; accepted 18 June 2003.

Address correspondence to Reto Crameri, Swiss Institute of Allergy and Asthma Research, Obere Strasse 22, CH-7270 Davos, Switzerland.e-mail: crameri@siaf.unizh.ch 